\title{
Icariin alters the expression of glucocorticoid receptor, FKBP5 and SGK1 in rat brains following exposure to chronic mild stress
}

\author{
KAI WEI $^{1 *}$, YIZHE XU $^{1,2^{*}}$, ZHENGXIAO ZHAO ${ }^{1,2}$, XIAO WU $^{1,2}$, YIJIE DU $^{1,2}$,

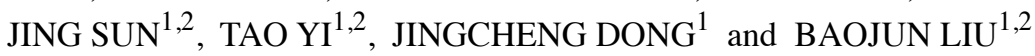 \\ ${ }^{1}$ Department of Integrative Medicine, Huashan Hospital, Fudan University, Shanghai 200040; \\ ${ }^{2}$ The Academy of Integrative Medicine of Fudan University, Shanghai 200032, P.R. China
}

Received November 26, 2015; Accepted April 27, 2016

DOI: $10.3892 / \mathrm{ijmm} .2016 .2591$

\begin{abstract}
Icariin, a flavonoid and a major constituent of Herba Epimedii, has been previously demonstrated to possess potential antidepressant-like effects. In the present study, we established a rat model of depression induced by unpredictable chronic mild stress (CMS) in order to examine the effects of icariin treatment. The rats were allocated into the control group or one of the treatment groups [exposure to CMS plus oral administration of saline, icariin (20 or $40 \mathrm{mg} / \mathrm{kg}$ ) or fluoxetine $(10 \mathrm{mg} / \mathrm{kg})]$. We examined the therapeutic effects of icariin administration on depression-like behaviors (with a sucrose preference test), on the mRNA and protein expression of glucocorticoid receptor (GR), FK506 binding protein 5 (FKBP5) and serum- and glucocorticoid-inducible kinase 1 (SGK1), as well as on the distribution of GR (in the cytoplasm and nucleus) in both the hippocampus and the prefrontal cortex following exposure to CMS. Our results revealed that the oral administration of icariin (20 and $40 \mathrm{mg} / \mathrm{kg}$ ) for 35 consecutive days attenuated the development of depression-like behaviors induced by exposure to CMS. The increased mRNA expression of GR and SGK1 in the prefrontal cortex was reversed by icariin treatment. Moreover, the CMS-induced increases in the levels of cytosolic GR and SGK1 were partially restored by icariin administration in both the hippocampus and the prefrontal cortex, particularly in the hippocampus. Icariin also partially reversed the upregulated epxression of nuclear GR in the prefrontal cortex and that of FKBP5 in the hippocampus. On the whole, our findings indicate that icariin may have therapeutic applications as a potential antidepressant with multiple targets in both the hippocampus and prefrontal cortex. It exerts antidepressant-like effects by
\end{abstract}

Correspondence to: Dr Baojun Liu, Department of Integrative Medicine, Huashan Hospital, Fudan University, 12 Middle Urumqi Road, Shanghai 200040, P.R. China

E-mail:1bj825@163.com

*Contributed equally

Key words: icariin, chronic mild stress, glucocorticoid receptor, FK506 binding protein 5, serum- and glucocorticoid-inducible kinase 1 , hypothalamic-pituitary-adrenal axis restoring the negative feedback regulation of the hypothalamicpituitary-adrenal axis, which is at least partially attributed to normalization of the distribution of GR, and decreases in the expression levels of FKBP5 and SGK1.

\section{Introduction}

The hypothalamic-pituitary-adrenal (HPA) axis is the major system involved in the stress response, and the dysregulation of the HPA axis is associated with depression (1). The activation of the HPA axis may lead to the release of glucocorticoid (GC) from the adrenal glands $(2,3)$. GCs regulate stress responses, including the successful adaptation to stress through the negative feedback regulation of the HPA axis by binding to the glucocorticoid receptor (GR) (4). Under pathological conditions, the impairment of the GR-mediated negative feedback system of the HPA axis results in constant HPA axis hyperactivity and chronically high GC levels, leading to the development of depressive disorders $(5,6)$. FK506 binding protein 5 (FKBP5), which promotes GR stability and reduces GR sensitivity to GC, is a negative modulator of GR activity that may inhibit the negative feedback loop of the HPA axis $(7,8)$. Serum- and glucocorticoid-inducible kinase 1 (SGK1) has also been implicated in the cellular stress response, as well as in neuronal function; it is a modulator of GC effects on neurogenesis and GR function, particularly in depression (9-11). The hippocampus and the prefrontal cortex are involved in the negative feedback regulation of the HPA axis and in the pathogenesis of depression $(1,4,12)$.

Icariin [2-(4'-methoxylphenyl)-3-rhamnosido-5-hydroxyl7-glucosido-8-(3'-methyl-2-butylenyl)-4-chromanone] (Fig. 1), a flavonoid, is a major constituent of Herba Epimedii, and exerts a wide range of pharmacological activities. Our previous studies demonstrated that icariin may potentially function as a novel antidepressant (13-15), which exerts an antidepressant effect by inhibiting neuroinflammation (15), protecting against corticosterone-induced apoptosis in rat hippocampal neurons (16), as well as by regulating the function of the HPA axis (14,17-19). In the present study, a chronic mild stress (CMS) protocol was used in order to establish a rat model of depression, which mimics many of the symptoms of depression in humans (20). We examined the therapeutic effects of icariin administration on depression-like behaviors, the mRNA and protein expression of GR, FKBP5 and SGK1, as well as the distribution of GR 
in the cytoplasm and the nucleus in both the hippocampus and the prefrontal cortex induced by exposure to CMS.

\section{Materials and methods}

Reagents. Icariin, [98.93\% purity, as verified by high-performance liquid chromatography (HPLC)] was purchased from the Shanghai Ronghe Medical Science Co., Ltd. (Shanghai, China). Fluoxetine was obtained from Eli Lilly Co. (Suzhou, China) and diluted in saline to a final concentration of $10 \mathrm{mg} / \mathrm{ml}$. A nuclear and cytoplasmic protein extraction kit, BCA kit and loading buffer were purchased from the Beyotime Institute of Biotechnology (Haimen, China). The rat corticosterone ELISA kit was obtained from eBioscience (San Diego, CA, USA). The primers for the nuclear receptor subfamily 3 , group $\mathrm{C}$, member 1 (glucocorticoid receptor) (Nr3c1), FKBP5, SGK1 and $\beta$-actin genes encoding rat GR, FKBP5, SGK1 and $\beta$-actin, respectively, were supplied by Sangon Technologies (Shanghai, China).

Animals and ethics approval. Fifty male Sprague-Dawley (SD) rats (5 weeks old, weighing 120-140 g) were purchased from the Shanghai Laboratory Animal Co. (SLAC; Shanghai, China). All the rats were individually housed in a laminar-flow room under specific pathogen-free (SPF) conditions and reared under the following conditions: an average room temperature of $22 \pm 1^{\circ} \mathrm{C}$, a relative humidity of $50-60 \%$, a $12 \mathrm{~h}$ light/dark cycle (lights on from 6:00 a.m. to 6:00 p.m.) and access to food and water ad libitum. The rats were allowed to acclimatize for at least 7 days prior to the commencement of the experiments. All experiments were conducted in accordance with the ethical standards of the Animal Care and Use Committee at Huashan Hospital of Fudan University (Shanghai, China). All efforts were made to minimize the number of animals used and their suffering.

Chronic stress paradigm and drug treatment. The CMS-exposed and the control groups were housed in separate rooms under similar standard conditions. The procedure for the induction of CMS consisted of once or twice daily exposure to various unpredictable mild stressors in a random order for 5 weeks according to our standard protocol (15), which may lead to a chronic depression-like state that develops gradually over time (21). The stressors included: food deprivation $(24 \mathrm{~h})$, overnight water deprivation $(18 \mathrm{~h})$ followed by $1 \mathrm{~h}$ of empty water bottle replacement, cage tilt $\left(45^{\circ}\right)$ for $18 \mathrm{~h}$, overnight illumination (60 W lamp) for $13 \mathrm{~h}$, soiled cage with $200 \mathrm{ml}$ water in $100 \mathrm{~g}$ sawdust bedding for $21 \mathrm{~h}$, forced swimming at $12^{\circ} \mathrm{C}$ for $5 \mathrm{~min}$, physical restraint in a clear plastic tube with air vents at the nasal end for $2 \mathrm{~h}$ and pair housing for $24 \mathrm{~h}$. The rats in the different groups received either the vehicle (saline $1 \mathrm{ml} / 100 \mathrm{~g}$ for both the control and CMS groups), icariin (20 and $40 \mathrm{mg}$ / $\mathrm{kg})$ or fluoxetine $(10 \mathrm{mg} / \mathrm{kg})$ by oral administration once daily for 35 days. The body weight of all the rats was weighed and recorded on the 1st and 35th days of the experiment using a platform scale. The behavioral tests were performed at least $16-18 \mathrm{~h}$ after the final treatment in order to avoid the acute effects of drug treatments.

Sucrose preference test (SPT). All the rats were initially trained to consume palatable sucrose solution $(1 \%, \mathrm{w} / \mathrm{v})$ by providing it

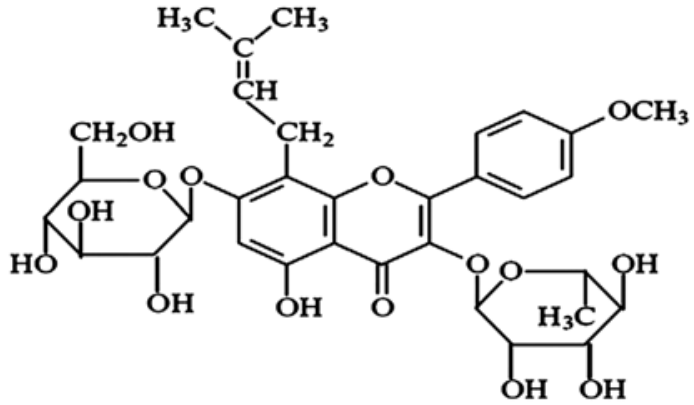

Figure 1. Chemical structure of icariin $\left(\mathrm{C}_{33} \mathrm{H}_{40} \mathrm{O}_{15}\right.$; molecular weight, 676.67).

Table I. Sequences of primers used for RT-qPCR.

\begin{tabular}{lcl}
\hline Gene & $\begin{array}{c}\text { GenBank } \\
\text { accession no. }\end{array}$ & \multicolumn{1}{c}{ Sequence $\left(5^{\prime} \rightarrow 3^{\prime}\right)$} \\
\hline Nr3c1 & NM_012576.2 & $\begin{array}{l}\text { F: GGGACACGAATGAGGATTG } \\
\text { R: CACACTGCTGGGACTTGAT }\end{array}$ \\
FKBP5 & NM_001012174.1 & $\begin{array}{l}\text { F: GTTCAGCTGTGCAATCCAGA } \\
\text { R: AGGGTGTTCTGTGCTCTTCAA }\end{array}$ \\
SGK1 & NM_001193568.1 & $\begin{array}{l}\text { F: GCCTGCCTCCGTCTACA } \\
\text { R: GCCTTGCTGAGTTGTGAT }\end{array}$ \\
$\beta$-actin & NM_031144.2 & $\begin{array}{l}\text { F: CCTCTATGCCAACACAGT } \\
\text { R: AGCCACCAATCCACACAG }\end{array}$ \\
\hline
\end{tabular}

FKBP5, FK506 binding protein 5; SGK1, serum- and glucocorticoid-inducible kinase $1 ; \mathrm{F}$, forward; $\mathrm{R}$, reverse.

as the only drinking fluid for $48 \mathrm{~h}$ in order to avoid neophobia. On the 36th day, all the rats were deprived of water for $23 \mathrm{~h}$ prior to the test. Sucrose preference was then determined by giving the rats a free choice for $1 \mathrm{~h}$ between 2 identical bottles placed in the cage and filled with either $1 \%$ sucrose solution or tap water. The SPT is used to determine deppressive states, as the animals are observed to examine their interest in seeking out a sweet rewarding drink as opposed to plain drinking water. The selection of the sweetened drink is typical of normal behaviour, and the non-selection of the sweetened drink is indicative of depression. Sucrose preference was defined as ( $\mathrm{ml}$ sucrose/total $\mathrm{ml}$ consumed) $\mathrm{x} 100 \%$.

Collection of brain tissue. In order to avoid fluctuations in hormone levels, all the rats were decapitated between 11:00 a.m. and 1:00 p.m. on the 37th day. The entire hippocampus and prefrontal cortex of each rat brain were rapidly dissected on ice, frozen in liquid nitrogen and stored at $-80^{\circ} \mathrm{C}$ for further analysis.

Reverse transcription-quantitative polymerase chain reaction $(R T-q P C R)$. Total RNA was extracted from the rat hippocampus and prefrontal cortex using TRIzol reagent followed by treatment with RNase-free DNase I (both from Invitrogen Life Technologies, Carlsbad, CA, USA). Reverse transcription was performed using the One-Step RNA-PCR kit (Takara, Dalian, China), according to the manufacturer's instructions. Quantitative (real-time) PCR was performed on a 
7300 Real-Time PCR system using SYBR-Green PCR Master Mix (Sangon Technologies). Following the addition of the primers (Table I) and template DNA in duplicate, PCR amplification was performed as follows: $95^{\circ} \mathrm{C}$ for $10 \mathrm{~min}, 40$ cycles of $60^{\circ} \mathrm{C}$ for $60 \mathrm{sec}$ and $95^{\circ} \mathrm{C}$ for $15 \mathrm{sec}$, and a melting curve from 60 to $95^{\circ} \mathrm{C}$ to ensure the amplification of a single product. The cycle passing threshold $(\mathrm{Ct})$ was recorded for each mRNA, and $\beta$-actin was used as the endogenous control for data normalization. The relative expression levels were calculated using the $2^{-\Delta \Delta \mathrm{Ct}}$ method.

Western blot analysis. The proteins were extracted from the rat hippocampus and prefrontal cortex using a nuclear and cytoplasmic protein extraction kit (Beyotime Institute of Biotechnology). The protein concentrations were quantified using the BCA method. The protein samples $(40-80 \mu \mathrm{g})$ were dissolved with an equal volume of loading buffer, separated on $10 \%$ SDS-PAGE and then electrotransferred at $90 \mathrm{~V}$ to PVDF membranes. The membranes were blocked with TBST containing 5\% non-fat milk for $1 \mathrm{~h}$ at room temperature followed by incubation with primary antibodies in the refrigerator overnight at $4^{\circ} \mathrm{C}$. The following primary antibodies were used: rabbit anti-GR (1:3,000, ab109022), rabbit anti-FKBP5 (1:3,000, ab2901), rabbit anti-SGK1 (1:1,000, ab59337) (all from Abcam, Cambridge, MA, USA) and rabbit anti- $\beta$-actin, rabbit anti-lamin B (1:1,000; Bioworld Technology Co., Ltd., Nanjing, China). The blots were washed extensively with TBST and incubated with secondary antibodies in TBST/5\% non-fat milk for $1 \mathrm{~h}$ at room temperature. Subsequently, the signal was detected using an enhanced chemiluminescence method (ECL kit; Millipore, Billerica, MA, USA). Finally, the membranes were imaged and analyzed using Quantity One Image Analysis Software (Syngene, Cambridge, UK).

Statistical analysis. Statistical analysis was performed using SPSS 20.0 software. All data in the figures are presented as the means \pm SD. One-way analysis of variance (ANOVA) was performed if the data followed a normal distribution and the variances were homogeneous; the least significant difference (LSD) test was used for further pairwise comparison. The non-parametric test was adopted if the data did not follow a normal distribution or the variances were not homogeneous; the Games-Howell test was used for further pairwise comparison. A value of $\mathrm{p}<0.05$ was considered to indicate a statistically significant difference.

\section{Results}

Effects of icariin on body weight and sucrose preference rate in rats exposed to CMS. Fig. 2A shows that the body weight of the rats exposed to CMS for 5 weeks was significantly lower than that of the control animals $(\mathrm{p}<0.01)$, and it was markedly increased by treatment with icariin $(40 \mathrm{mg} / \mathrm{kg})$ and fluoxetine $(10 \mathrm{mg} / \mathrm{kg})$ compared with the CMS group $(\mathrm{p}<0.01)$. The body weight of the rats in the fluoxetine group was higher than that in the icariin $(40 \mathrm{mg} / \mathrm{kg})$ group $(\mathrm{p}<0.05)$ on day 35. Moreover, as shown in Fig. 2B, the rats exposed to CMS for 5 weeks displayed a significantly reduced preference for sucrose solution $(\mathrm{p}<0.01)$ compared with the control rats. Treatment with icariin $(20$ and $40 \mathrm{mg} / \mathrm{kg})$ and fluoxetine $(10 \mathrm{mg} / \mathrm{kg})$ reversed the
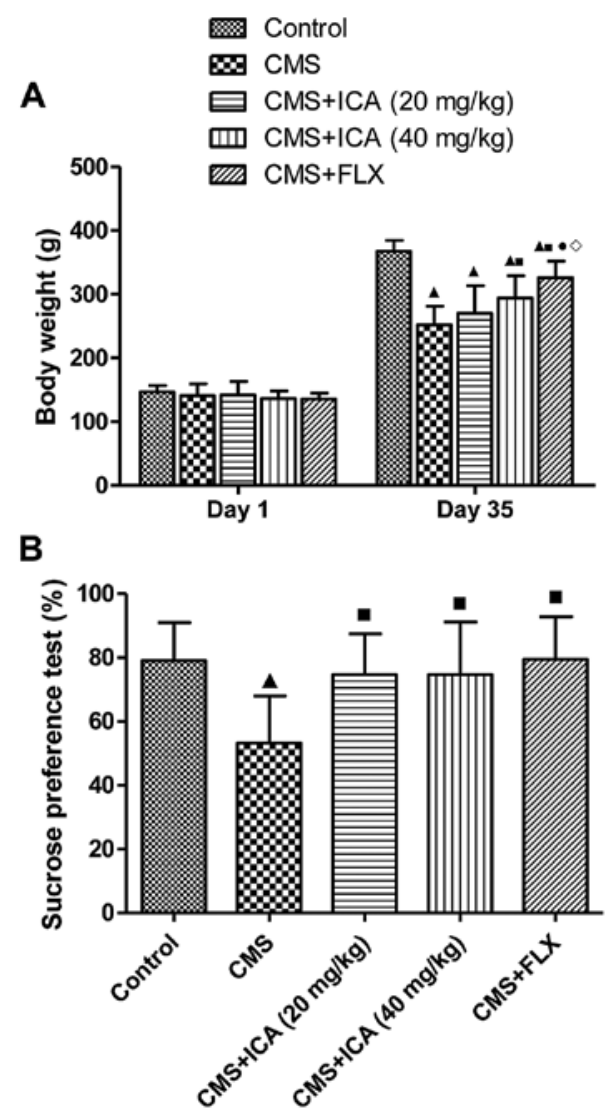

Figure 2. Icariin treatment attenuates depression-like behaviors in a rat model of depression induced by CMS. (A) Effects of icariin on the body weight of CMS-exposed rats. These data show body weight on days 1 and 35. (B) Effects of icariin on the sucrose preference of CMS-exposed rats. Data represent the means $\pm \mathrm{SD}, \mathrm{n}=10$ rats/group for body weight, $\mathrm{n}=8-10$ rats/group for the sucrose preference test. ${ }^{\wedge} \mathrm{p}<0.01$ vs. control; $" \mathrm{p}<0.01$ vs. CMS; ${ }^{\bullet} \mathrm{p}<0.01$ vs. CMS + ICA $(20 \mathrm{mg} / \mathrm{kg}) ;{ }^{\diamond} \mathrm{p}<0.05 \mathrm{vs} . \mathrm{CMS}+\mathrm{ICA}(40 \mathrm{mg} / \mathrm{kg})$. CMS,chronic mild stress; ICA, icariin; FLX, fluoxetine.

CMS-induced reduction in sucrose preference $(\mathrm{p}<0.01)$. Thus, icariin may be useful in reducing depression-like behaviours.

Effects of icariin on mRNA Nr3cl expression and on cytosolic and nuclear GR levels in the hippocampus and prefrontal cortex of rats exposed to CMS. As shown in Fig. 3A and B, exposure to CMS slightly decreased the mRNA expression of $\mathrm{Nr} 3 \mathrm{cl}$ in the hippocampus ( $>>0.05)$; however, the mRNA expression of $\mathrm{Nr} 3 \mathrm{cl}$ was significantly increased in the prefrontal cortex $(\mathrm{p}<0.01)$ of rats exposed to CMS, compared with the rats in the control group. Following treatment with icariin (20 and $40 \mathrm{mg} / \mathrm{kg})$ and fluoxetine $(10 \mathrm{mg} / \mathrm{kg})$, the mRNA expression of $\mathrm{Nr} 3 \mathrm{cl}$ in the prefrontal cortex was markedly decreased $(\mathrm{p}<0.01$; levels were similar to those of control group). Compared with the control group, the expression of cytosolic GR was significantly upregulated by exposure to CMS in both the hippocampus and the prefrontal cortex $(\mathrm{p}<0.05$ and $\mathrm{p}<0.01$, respectively; Fig. $3 \mathrm{C}$ and D), whereas treatment with icariin (20 and $40 \mathrm{mg} / \mathrm{kg}$ ) and fluoxetine $(10 \mathrm{mg} / \mathrm{kg})$ significantly decreased the expression of cytosolic GR in the hippocampus $(\mathrm{p}<0.01$, $\mathrm{p}<0.01$ and $\mathrm{p}<0.05$, respectively), compared with the CMS group. The upregulated cytosolic GR expression in the prefrontal cortex was only downregulated by icariin $(20 \mathrm{mg} / \mathrm{kg})(\mathrm{p}<0.01)$, whereas the expression level was further increased in the icariin 

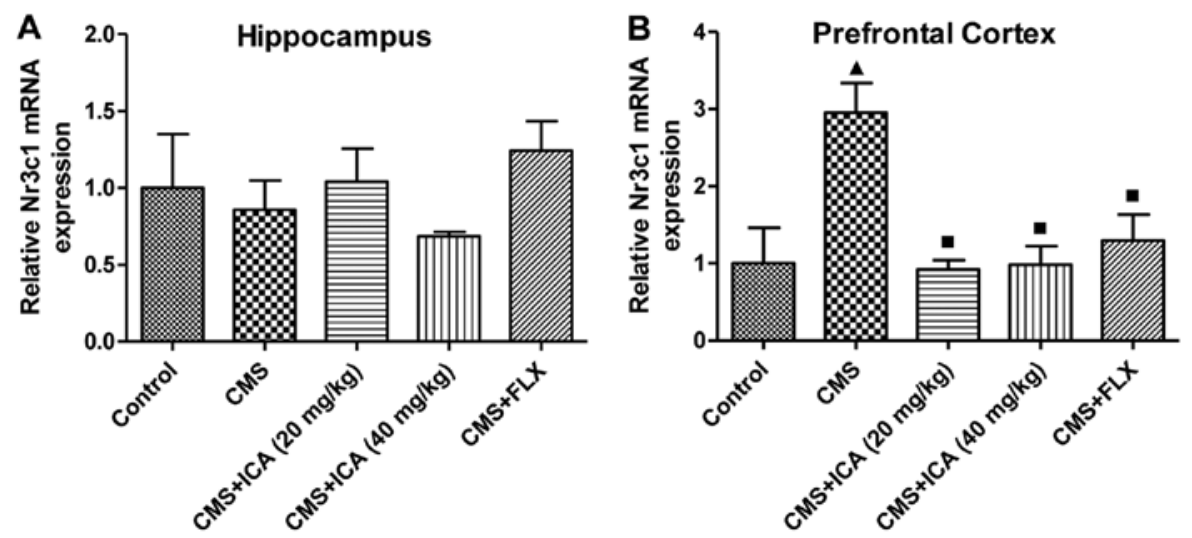

C

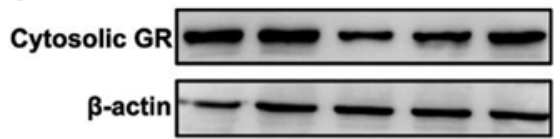

D
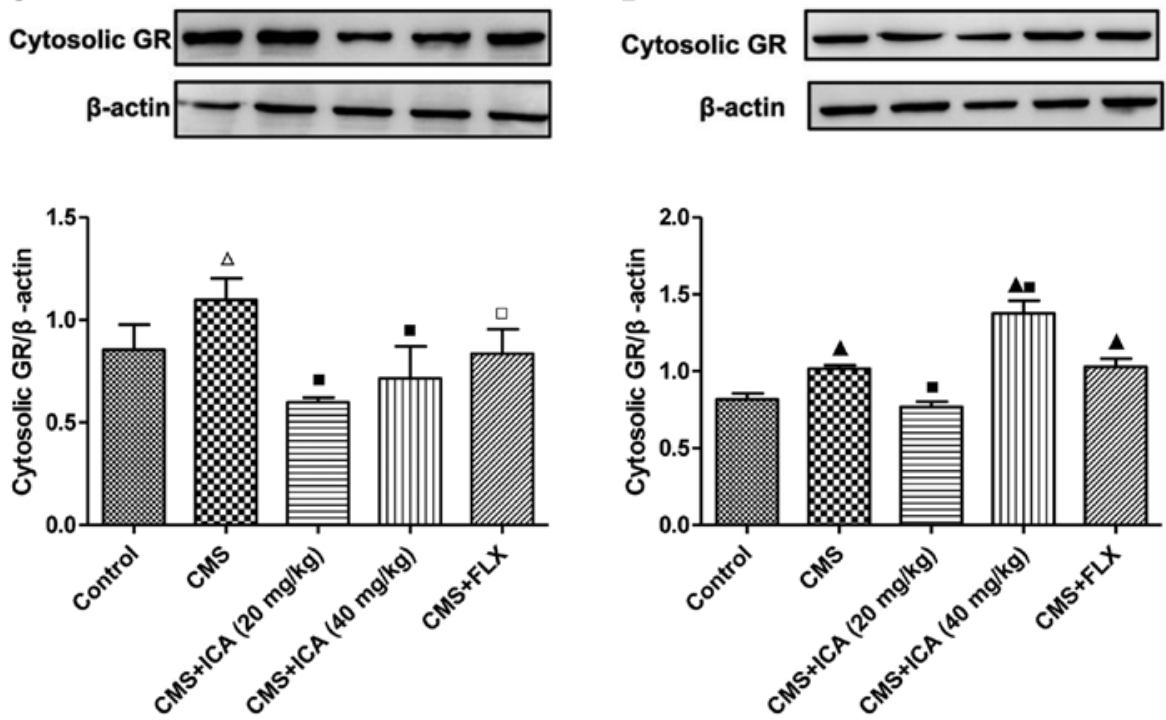

E

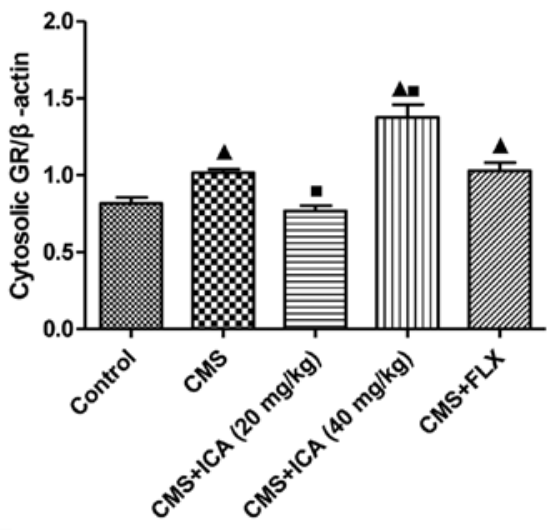

Nuclear GR $\square$

$\mathbf{F}$
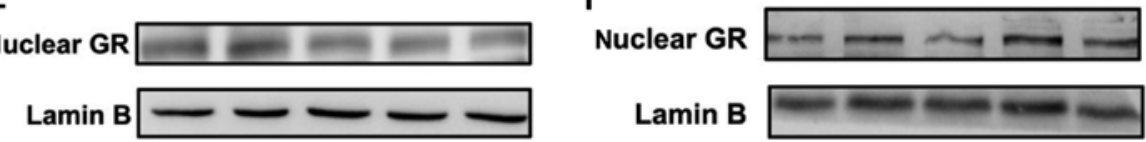

Lamin B
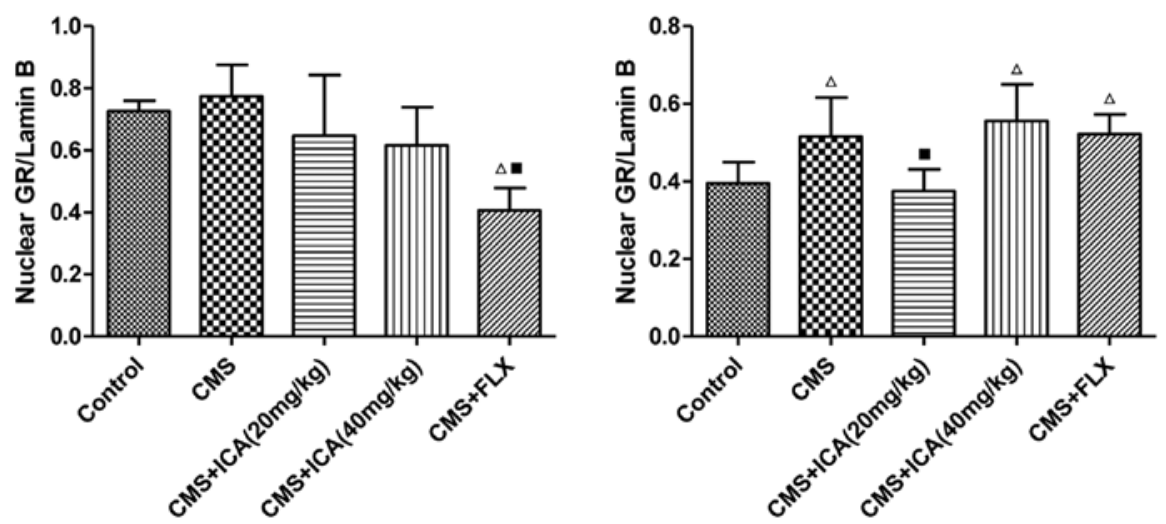

Figure 3. Icariin treatment normalizes the mRNA expression of Nr3cl and GR distribution in the hippocampus and the prefrontal cortex of CMS-exposed rats. (A) CMS induced a slight decrease in the mRNA expression of $\mathrm{Nr} 3 \mathrm{cl}$ in the hippocampus and (B) a significant increase in the mRNA expression of $\mathrm{Nr} 3 \mathrm{c} 1$ in the prefrontal cortex, which was significantly decreased by icariin. (C and D) CMS induced an increase in cytosolic GR expression in the hippocampus and the prefrontal cortex, and icariin reduced the accumulation of cytosolic GR in the hippocampus and in the prefrontal cortex at a dose of $20 \mathrm{mg} / \mathrm{kg}$. (E and F) Nuclear GR expression was only upregulated by exposure to CMS in the prefrontal cortex, and icariin $(20 \mathrm{mg} / \mathrm{kg})$ reversed this effect. Data represent the means $\pm \mathrm{SD}$, $\mathrm{n}=3$ rats/group. ${ }^{\mathrm{p}} \mathrm{p}<0.01$ and ${ }^{\triangle} \mathrm{p}<0.05$ vs. control; ${ }^{\mathrm{p}} \mathrm{p}<0.01$ and ${ }^{\square} \mathrm{p}<0.05$ vs. CMS. Nuclear receptor subfamily 3 , group $\mathrm{C}$, member 1 (glucocorticoid receptor), $\mathrm{Nr} 3 \mathrm{c} 1$; CMS, chronic mild stress; ICA, icariin; FLX, fluoxetine; GR, glucocortcoid receptor.

$(40 \mathrm{mg} / \mathrm{kg})$ group $(\mathrm{p}<0.01)$. As demonstrated in Fig. 3E and F, nuclear GR expression in the hippocampus was not affected by exposure to CMS ( $p>0.05$ ); however, it was upregulated in the prefrontal cortex by exposure to CMS $(\mathrm{p}<0.05)$, compared with the control group. Treatment with icariin $(20 \mathrm{mg} / \mathrm{kg})$ significantly reduced the distribution of nuclear GR in the prefrontal 

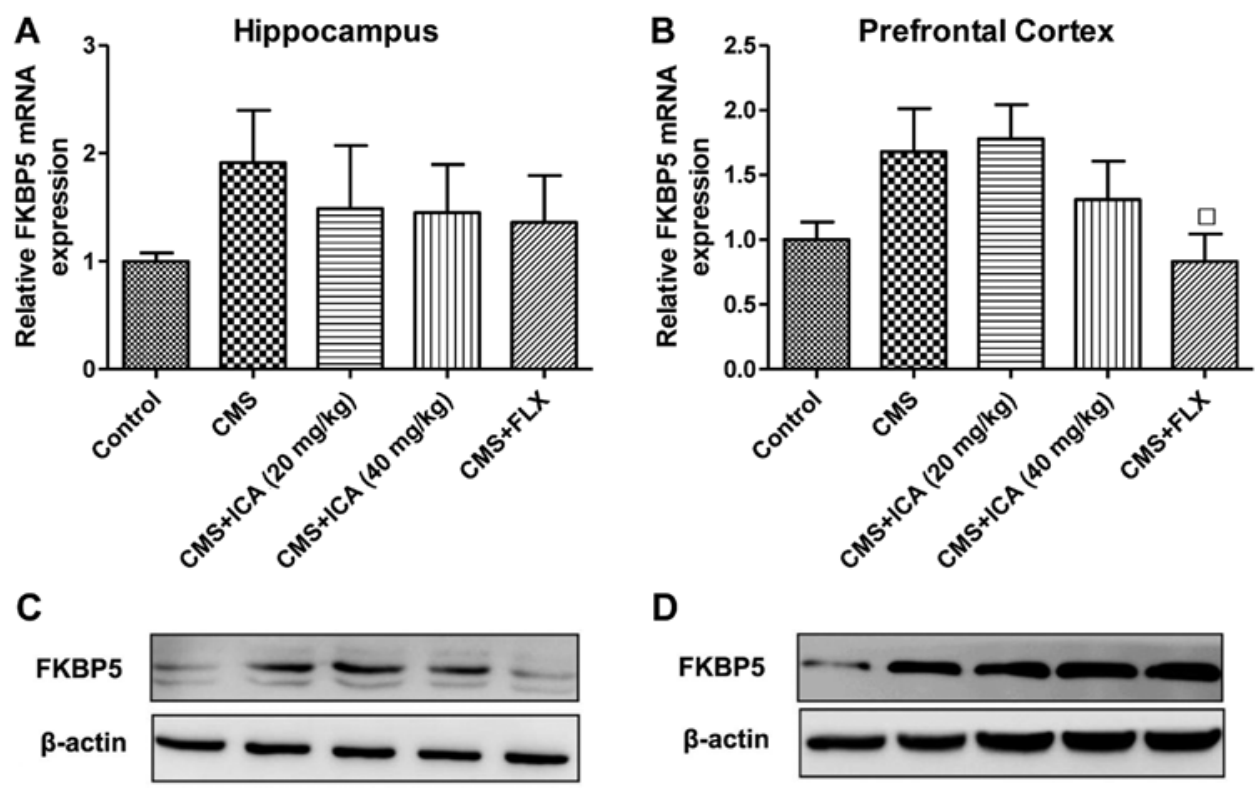

D
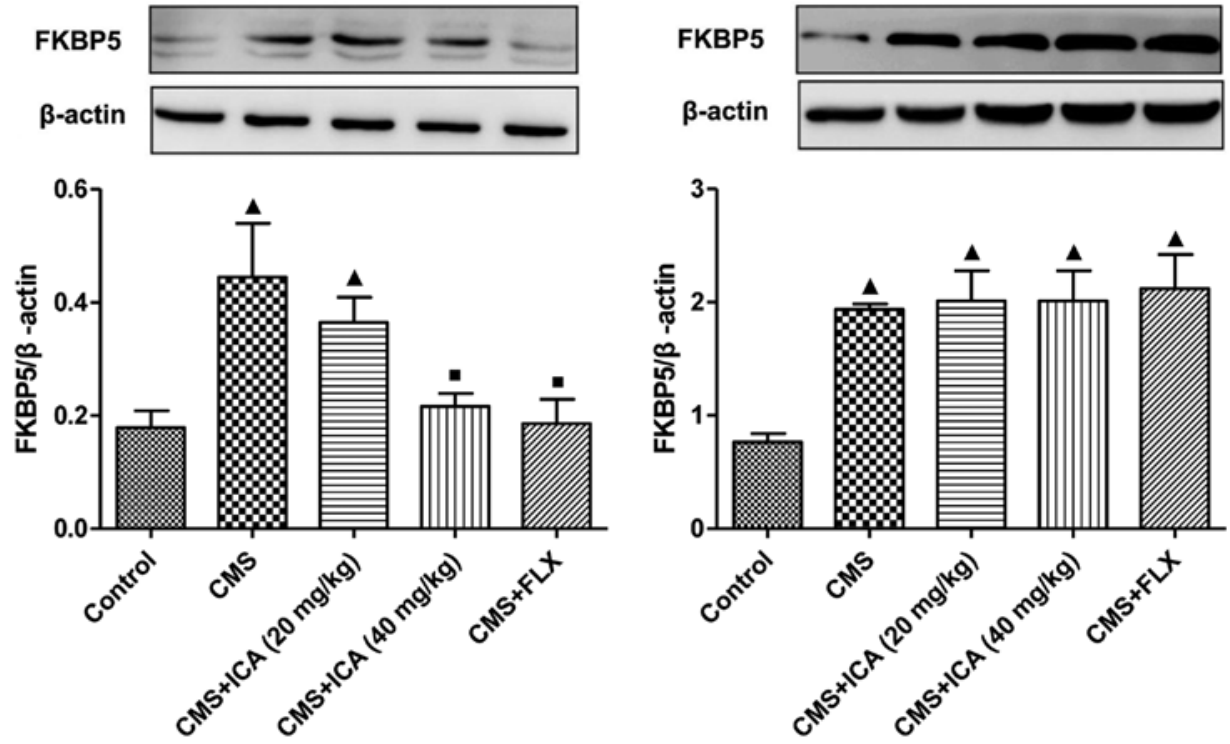

Figure 4. Icariin treatment (40mg/kg) abrogates the increase in the protein expression of FKBP5 in the hippocampus of CMS-exposed rats. (A and B) exposure to CMS had no effect on the mRNA expression of FKBP5 in the hippocampus and the prefrontal cortex of rats. (C and D) CMS induced an increase in FKBP5 protein expression in the hippocampus and the prefrontal cortex, and icariin treatment $(40 \mathrm{mg} / \mathrm{kg}$ ) abrogated the upregulation in the expression of FKBP5 in the hippocampus. Data represent the means $\pm \mathrm{SD}, \mathrm{n}=3$ rats/group. ${ }^{\wedge} \mathrm{p}<0.01$ vs. control; ${ }^{\mathrm{p}} \mathrm{p}<0.01$ and ${ }^{\square} \mathrm{p}<0.05$ vs. CMS. FKBP5, FK506 binding protein 5; CMS, chronic mild stress; ICA, icariin; FLX, fluoxetine.

cortex ( $\mathrm{p}<0.01)$, compared with the CMS group, whereas icariin $(40 \mathrm{mg} / \mathrm{kg})$ and fluoxetine $(10 \mathrm{mg} / \mathrm{kg})$ had no effect on nuclear GR expression in the prefrontal cortex.

Effects of icariin on the expression of FKBP5 in the hippocampus and prefrontal cortex of rats exposed to CMS. As shown in Fig. 4, the mRNA expression of FKBP5 was increased (although not signifiantly) in both regions of the brain following exposure to CMS ( $p>0.05$ ), whereas the protein expression of FKBP5 was significantly upregulated $(\mathrm{p}<0.01)$ in both the hippocampus and the prefrontal cortex of the rats in the CMS group, compared with those in the control group. Treatment with icariin $(40 \mathrm{mg} / \mathrm{kg})$ and fluoxetine $(10 \mathrm{mg} / \mathrm{kg})$ markedly abrogated the upregulation in the expression of FKBP5 in the hippocampus $(\mathrm{p}<0.01$; the levels were close to those of the control group), whereas they had no effect on FKBP5 protein expression in the prefrontal cortex. Moreover, fluoxetine $(10 \mathrm{mg} / \mathrm{kg})$ also significantly downregulated the mRNA expression of FKBP5 in the prefrontal cortex $(p<0.05)$, compared with the CMS group.
Effects of icariin on the expression of SGK1 in the hippocampus and prefrontal cortex of rats exposed to CMS. As shown in Fig. 5A and B, the mRNA expression of SGK1 was significantly increased $(\mathrm{p}<0.01)$ in the prefrontal cortex of the CMS-exposed rats, whereas there was no significant difference in the hippocampus, compared with the control group rats. Following treatment with icariin (20 and $40 \mathrm{mg} / \mathrm{kg})$ and fluoxetine $(10 \mathrm{mg} / \mathrm{kg})$, the mRNA expression of SGK1 was markedly decreased in the prefrontal cortex $(\mathrm{p}<0.01$; levels were close to those of the control group), and was also decreased in the hippocampus $(\mathrm{p}<0.05, \mathrm{p}<0.01$ and $\mathrm{p}<0.01$, respectively), compared with the CMS group. As shown in Fig. $5 \mathrm{C}$ and D, the protein expression of SGK1 was upregulated by exposure to CMS in both the hippocampus and prefrontal cortex $(p<0.01)$, as compared with the control group. Treatment with icariin (20 and $40 \mathrm{mg} / \mathrm{kg}$ ) and fluoxetine $(10 \mathrm{mg} / \mathrm{kg})$ significantly decreased the expression of SGK1 in the hippocampus $(\mathrm{p}<0.01)$, and in the prefrontal cortex following treatment with the higher dose of icariin and fluoxetine ( $<<0.05$ for $40 \mathrm{mg} / \mathrm{kg}$ icariin, $\mathrm{p}<0.01$ for $10 \mathrm{mg} / \mathrm{kg}$ fluoxetine), compared with the CMS group. 

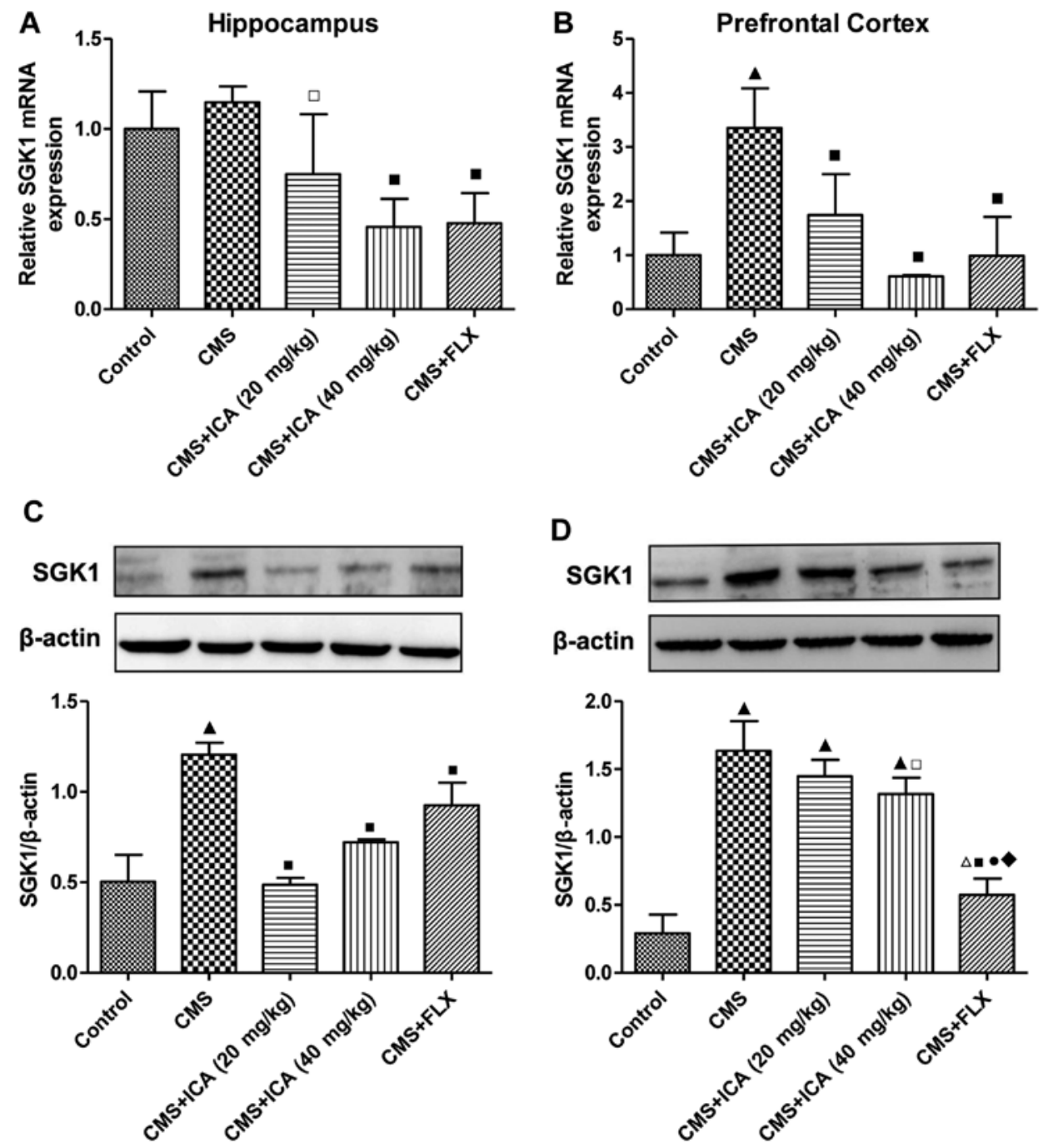

Figure 5. Icariin treatment reduces the upregulation in the mRNA and protein expression of SGK1 in the hippocampus and the prefrontal cortex of CMS-exposed rats. (A) There was no significant increase in the mRNA expression of SGK1 in the hippocampus whereas (B) SGK1 levels were upregulated in the prefrontal cortex of the CMS rats, and icariin markedly normalized its expression. (C and D) The protein expression of SGK1 was increased by exposure to CMS in the hippocampus and the prefrontal cortex, and icariin reduced the upregulated protein expression of SGK1 in both brain regions, particularly in the hippocampus.

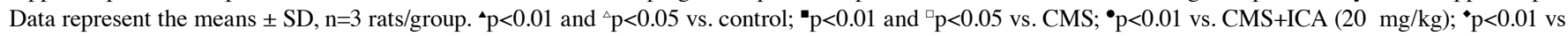
CMS + ICA (40 mg/kg). Serum- and glucocorticoid-inducible kinase 1,SGK1; CMS, chronic mild stress; ICA, icariin; FLX, fluoxetine.

Furthermore, fluoxetine $(10 \mathrm{mg} / \mathrm{kg})$ decreased SGK1 expression in the prefrontal cortex to a lower level than in the icariin treatment groups $(\mathrm{p}<0.01)$.

\section{Discussion}

Upon binding to GC, the GR undergoes a conformational change, which allows it to translocate to the nucleus (7). In the nucleus, the GR binds to glucocorticoid response elements (GRE) on the target DNA as a positive or negative transcription factor in order to regulate the transcription of GR-responsive genes $(12,22,23)$. The decreased mRNA expression of GR has been found in the frontal cortex of post-mortem tissue from patients with schizophrenia and mood disorders (24), and in the hippocampus in rodent models of depression induced by social defeat (13) or CMS $(25,26)$, as well as in the hippocampus and the prefrontal cortex of mice receiving chronic dexamethasone treatment (27). Moreover, it has been demonstrated that the cytosolic GR levels in the ventral hippocampus and the prefrontal cortex are significantly increased following exposure to CMS, and they may be completely normalized (mainly in the prefrontal cortex) by the administration of duloxetine (1). Previous research has also demonstrated that the novel antidepressant icaritin exerted therapeutic effects by increasing the downregulated mRNA expression of GR in the hippocampus of socially-defeated mice (13); emodin opposed depression-like behaviors in CMS-exposed mice by upregulating the mRNA expression of hippocampal GR (26). In the present study, the GR mRNA expression in the hippocampus was slightly decreased by exposure to CMS; however, this effect was not significant, which is a similar trend to that observed in previous research $(25,26)$. Notably, in contrast to the findings of Skupio et al (27), in this study, the GR mRNA expression was markedly upregulated by exposure to CMS in the prefrontal cortex, which was decreased by icariin and fluoxetine treatment. Cytosolic GR expression in both the hippocampus and the prefrontal cortex was increased by exposure to CMS, as has been demonstrated by previous research (1), which indicated decreases in GC sensitivity of the 
GR in these brain regions. Icariin and fluoxetine normalized the increased expression of cytosolic GR in hippocampus, and only icariin $(20 \mathrm{mg} / \mathrm{kg})$ downregulated the expression of cytosolic GR in the prefrontal cortex, which was unexpectedly upregulated by icariin $(40 \mathrm{mg} / \mathrm{kg})$. The expression of nuclear GR in the hippocampus exhibited no obvious change following exposure to CMS, although fluoxetine decreased its expression. In the prefrontal cortex, exposure to CMS upregulated the expression of nuclear GR, which was only decreased by icariin at $20 \mathrm{mg} / \mathrm{kg}$, but not icariin at $40 \mathrm{mg} / \mathrm{kg}$ or fluoxetine. Previous research has confirmed that GR mitochondrial translocation also exists in the hippocampus and cortex of rats (28), and chronic high-dose corticosterone treatment may decrease the GR levels in the mitochondria in both primary cortical neurons and rodent prefrontal cortex (29). As serum corticosterone levels were upregulated in CMS-exposed rats in this study (data not shown), decreased mitochondrial GR in the hippocampus may also exist, which may be one reason for the slight decrease in the GR mRNA and the increased level of cytosolic GR in the hippocampus of the CMS-exposed rats. Following exposure to CMS in the present study, the observed increase in GR mRNA expression in the prefrontal cortex was contrary to previous findings (27). Moreover, the increased levels of cytosolic and nuclear GR in the prefrontal cortex were not in line with the increase in GR mRNA (2-3-fold increase). Further studies are warranted in order to explore the reason for these unexpected results. In the prefrontal cortex, the opposing effects of icariin $(20 \mathrm{mg} / \mathrm{kg})$ and icariin $(40 \mathrm{mg} / \mathrm{kg})$ on the level of cytosolic GR, as well as the unaltered level of nuclear GR in the icariin (40 $\mathrm{mg} / \mathrm{kg}$ ) group may indicate that the antidepressant-like effects exerted by icariin are not strictly dose-dependent.

As a negative modulator of GR activity, the mRNA and protein expression of FKBP5 may be induced by GR activation (7). When FKBP5 is bound to GRs, the GR has a lower binding affinity for GC and is retained in the cytoplasm. FKBP5 also enhances the stability of the GR, potentially protecting it from proteolysis, and reduces GR sensitivity to GCs (7). It has been found that CMS or chronic dexamethasone treatment may increase the mRNA and/or protein expression of FKBP5 in the brain of rodents, particularly in the hippocampus and the prefrontal cortex $(1,27,30,31)$. The upregulation of the mRNA and protein expression of FKBP5 in the frontal cortex has also been associated with the depression status (3). Antidepressants may significantly reverse increases in the mRNA and protein expression of FKBP5 in the hippocampus and/or prefrontal cortex of CMS-exposed rats (1,31). In line with previous research, this study found that exposure to CMS had a tendency to increase the mRNA expression of FKBP5, but markedly upregulated FKBP5 protein expression in the hippocampus and the prefrontal cortex. Icariin $(40 \mathrm{mg} / \mathrm{kg})$ and fluoxetine reversed the CMS-induced increase in FKBP5 protein expression in the hippocampus, whereas the protein expression of FKBP5 in the prefrontal cortex was not affected by either icariin or fluoxetine, which provides evidence for the specific targets of icariin in the treatment of depression.

SGK1, another GR target gene, which may be induced at both the mRNA and protein levels by GC, may act as a modulator of GC for neurogenesis and GR function. Therefore, it is involved in the pathogenesis of depression and may serve as a target for antidepressants $(9-11,32)$. It has been demonstrated that SGK1 is involved in the GC-induced reduction of the proliferation and differentiation of human hippocampal progenitor cells, by acting both downstream of GR activation (through SGK1-dependent inhibition of the Hedgehog pathway) and upstream of GR activation (through SGK1-dependent GR phosphorylation and nuclear translocation) (11). Hedgehog signaling promotes neuronal differentiation (33), and therefore the SGK1-dependent inhibition of this pathway results in decreased neurogenesis. The mRNA expression of SGK1 has been found to be significantly increased (positive correlation with mRNA expression of FKBP5) in the peripheral blood of drug-free depressed patients, as well as in the hippocampus of rats subjected to CMS (11). In addition, GC treatment clearly increased the expression of SGK1 in human neural stem cells, as did chronic restraint in the hippocampus and the prefrontal cortex in rodent models of depression $(27,34)$. Consistently, in the present study, exposure to CMS significantly increased the mRNA expression of SGK1 in the prefrontal cortex, and the protein expression of SGK1 in both the hippocampus and the prefrontal cortex. In the hippocampus, there was a slight increase in the mRNA expression of SGK1 following exposure to CMS; however, this effect was not significant. The administration of icariin and fluoxetine markedly suppressed the increase in the mRNA expression of SGK1 in the prefrontal cortex and the protein expression of SGK1 in both the hippocampus and the prefrontal cortex, among which fluoxetine decreased SGK1 to a lower level than icariin did in the prefrontal cortex. As regards the mRNA and protein expression of SGK1 in the prefrontal cortex, dose-dependent effects were observed between the icariin treatment groups. In addition, the increased mRNA and protein expression of SGK1 in the hippocampus and/or prefrontal cortex following exposure to CMS revealed that SGK1 may mainly participate in the downstream activation of GR in this study, which suggests that the inhibition of the Hedgehog pathway may also play a role in this model of experimental depression induced by CMS. Further studies are warranted in order to confirm this hypothesis.

To the best of our knowledge, this is the first study to demonstrate that icariin treatment may reverse depression-like behaviors in rodent models by downregulating the expression of FKBP5 and SGK1 in the hippocampus and/or the prefrontal cortex, and by normalizing the GR distribution between the cytoplasm and the nucleus, which are similar to the effects of fluoxetine to a certain degree. These data extend the mechanisms of icariin in treating depression, and provide molecular evidence that icariin may serve as a potentially effective antidepressant with specific targets in different regions of the brain, which may aid in the development of novel pharmacotherapeutic approaches that selectively target these important molecules. However, further experimental research with more samples, as well as large-scale randomized controlled clinical trials are necessary in the future in order to elucidate the molecular mechanisms underlying the pathogenesis of depression, and to examine in detail the mechanisms responsible for the antidepressant-like effects of icariin from experimental and clinical perspectives.

In conclusion, the present study demonstrated that the antidepressant-like effects of icariin are at least partially attributed to the normalization of GR distribution between the cytoplasm 
and the nucleus, as well as to decreases in the expression of FKBP5 and SGK1 in the hippocampus and/or prefrontal cortex, which may restore the normal negative feedback regulation of the HPA axis and normal neurogenesis in related brain regions.

\section{Acknowledgements}

The present study was funded by grants from the National Natural Science Foundation of China (nos. 81102562 and 81173390), the National Basic Science Program of China (no. 2009CB523000), the Chinese Ministry of Education Fund for Doctor Discipline Scientific Research (no. 20110071120072), and the Development Project of Shanghai Peak Disciplines-Integrative Medicine.

\section{References}

1. Guidotti G, Calabrese F, Anacker C, Racagni G, Pariante CM and Riva MA: Glucocorticoid receptor and FKBP5 expression is altered following exposure to chronic stress: modulation by antidepressant treatment. Neuropsychopharmacology 38: 616-627, 2013.

2. McEwen BS, Gould EA and Sakai RR: The vulnerability of the hippocampus to protective and destructive effects of glucocorticoids in relation to stress. Br J Psychiatry Suppl 15: 18-23, 1992.

3. Tatro ET, Everall IP, Kaul M and Achim CL: Modulation of glucocorticoid receptor nuclear translocation in neurons by immunophilins FKBP51 and FKBP52: implications for major depressive disorder. Brain Res 1286: 1-12, 2009.

4. Adzic M,Djordjevic J, Djordjevic A, Niciforovic A, Demonacos C, Radojcic $\mathrm{M}$ and Krstic-Demonacos M: Acute or chronic stress induce cell compartment-specific phosphorylation of glucocorticoid receptor and alter its transcriptional activity in Wistar rat brain. J Endocrinol 202: 87-97, 2009.

5. Sapolsky RM, Romero LM and Munck AU: How do glucocorticoids influence stress responses? Integrating permissive, suppressive, stimulatory, and preparative actions. Endocr Rev 21: 55-89, 2000.

6. Pariante CM: Risk factors for development of depression and psychosis. Glucocorticoid receptors and pituitary implications for treatment with antidepressant and glucocorticoids. Ann NY Acad Sci 1179: 144-152, 2009.

7. Binder EB: The role of FKBP5, a co-chaperone of the glucocorticoid receptor in the pathogenesis and therapy of affective and anxiety disorders. Psychoneuroendocrinology 34 (Suppl 1): S186-S195, 2009.

8. Wochnik GM, Rüegg J, Abel GA, Schmidt U, Holsboer F and Rein T: FK506-binding proteins 51 and 52 differentially regulate dynein interaction and nuclear translocation of the glucocorticoid receptor in mammalian cells. J Biol Chem 280: 4609-4616, 2005.

9. Miyata S, Koyama Y, Takemoto K, Yoshikawa K, Ishikawa T, Taniguchi M, Inoue K, Aoki M, Hori O, Katayama T and Tohyama M: Plasma corticosterone activates SGK1 and induces morphological changes in oligodendrocytes in corpus callosum. PLoS One 6: e19859, 2011.

10. Lang F, Strutz-Seebohm N, Seebohm G and Lang UE: Significance of SGK1 in the regulation of neuronal function. J Physiol 588: 3349-3354, 2010.

11. Anacker C, Cattaneo A, Musaelyan K, Zunszain PA, Horowitz M, Molteni R, Luoni A, Calabrese F, Tansey K, Gennarelli M, et al: Role for the kinase SGK1 in stress, depression, and glucocorticoid effects on hippocampal neurogenesis. Proc Natl Acad Sci USA 110: 8708-8713, 2013.

12. Djordjevic A, Adzic M, Djordjevic J and Radojcic MB: Stress type dependence of expression and cytoplasmic-nuclear partitioning of glucocorticoid receptor, hsp90 and hsp70 in Wistar rat brain. Neuropsychobiology 59: 213-221, 2009.

13. Wu X, Wu J, Xia S, Li B and Dong J: Icaritin opposes the development of social aversion after defeat stress via increases of GR mRNA and BDNF mRNA in mice. Behav Brain Res 256: 602-608, 2013

14. Li B, Duan X, Xu C, Wu J, Liu B, Du Y, Luo Q, Jin H, Gong W and Dong J: Icariin attenuates glucocorticoid insensitivity mediated by repeated psychosocial stress on an ovalbumin-induced murine model of asthma. Int Immunopharmacol 19: 381-390, 2014.
15. Liu B, Xu C, Wu X, Liu F, Du Y, Sun J, Tao J and Dong J: Icariin exerts an antidepressant effect in an unpredictable chronic mild stress model of depression in rats and is associated with the regulation of hippocampal neuroinflammation. Neuroscience 294: 193-205, 2015.

16. Liu B, Zhang H, Xu C, Yang G, Tao J, Huang J, Wu J, Duan X, Cao Y and Dong J: Neuroprotective effects of icariin on corticosterone-induced apoptosis in primary cultured rat hippocampal neurons. Brain Res 1375: 59-67, 2011.

17. Pan Y, Wang FM, Qiang LQ, Z Zhang DM and Kong LD: Icariin attenuates chronic mild stress-induced dysregulation of the LHPA stress circuit in rats. Psychoneuroendocrinology 35: 272-283, 2010.

18. Pan Y, Zhang WY, Xia X and Kong LD: Effects of icariin on hypothalamic-pituitary-adrenal axis action and cytokine levels in stressed Sprague-Dawley rats. Biol Pharm Bull 29: 2399-2403, 2006.

19. Pan Y, Kong LD, Li YC, Xia X, Kung HF and Jiang FX: Icariin from Epimedium brevicornum attenuates chronic mild stress-induced behavioral and neuroendocrinological alterations in male Wistar rats. Pharmacol Biochem Behav 87: 130-140, 2007.

20. Hill MN, Hellemans KG, Verma P, Gorzalka BB and Weinberg J: Neurobiology of chronic mild stress: parallels to major depression. Neurosci Biobehav Rev 36: 2085-2117, 2012.

21. Calabrese F, Guidotti G, Molteni R, Racagni G, Mancini M and Riva MA: Stress-induced changes of hippocampal NMDA receptors: modulation by duloxetine treatment. PLoS One 7: e37916, 2012

22. Maccari S, Mormède P, Piazza PV, Simon H, Angelucci L and Le Moal M: Hippocampal type I and type II corticosteroid receptors are modulated by central noradrenergic systems. Psychoneuroendocrinology 17: 103-112, 1992.

23. Pavlides C, Watanabe Y, Magariños AM and McEwen BS: Opposing roles of type I and type II adrenal steroid receptors in hippocampal long-term potentiation. Neuroscience 68: 387-394, 1995.

24. Webster MJ, Knable MB, O'Grady J, Orthmann J and Weickert CS: Regional specificity of brain glucocorticoid receptor mRNA alterations in subjects with schizophrenia and mood disorders. Mol Psychiatry 7: 985-994, 924, 2002.

25. Zheng H, Liu Y, Li W, Yang B, Chen D, Wang X, Jiang Z, Wang $H$, Wang $Z$, Cornelisson $G$ and Halberg F: Beneficial effects of exercise and its molecular mechanisms on depression in rats. Behav Brain Res 168: 47-55, 2006.

26. Li M, Fu Q, Li Y, Li S, Xue J and Ma S: Emodin opposes chronic unpredictable mild stress induced depressive-like behavior in mice by upregulating the levels of hippocampal glucocorticoid receptor and brain-derived neurotrophic factor. Fitoterapia 98: $1-10,2014$.

27. Skupio U, Tertil M, Sikora M, Golda S, Wawrzczak-Bargiela A and Przewlocki R: Behavioral and molecular alterations in mice resulting from chronic treatment with dexamethasone: relevance to depression. Neuroscience 286: 141-150, 2015.

28. Moutsatsou P, Psarra AM, Tsiapara A, Paraskevakou H, Davaris P and Sekeris CE: Localization of the glucocorticoid receptor in rat brain mitochondria. Arch Biochem Biophys 386: 69-78, 2001.

29. Du J, Wang Y, Hunter R, Wei Y, Blumenthal R, Falke C, Khairova R, Zhou R, Yuan P, Machado-Vieira R, et al: Dynamic regulation of mitochondrial function by glucocorticoids. Proc Natl Acad Sci USA 106: 3543-3548, 2009.

30. Xing Y, He J, Hou J, Lin F, Tian J and Kurihara H: Gender differences in CMS and the effects of antidepressant venlafaxine in rats. Neurochem Int 63: 570-575, 2013.

31. Xing Y, Hou J, Meng Q, Yang M, Kurihara H and Tian J: Novel antidepressant candidate RO-05 modulated glucocorticoid receptors activation and FKBP5 expression in chronic mild stress model in rats. Neuroscience 290: 255-265, 2015.

32. Webster MK, Goya L, Ge Y, Maiyar AC and Firestone GL: Characterization of sgk, a novel member of the serine/threonine protein kinase gene family which is transcriptionally induced by glucocorticoids and serum. Mol Cell Biol 13: 2031-2040, 1993.

33. Cai C, Thorne J and Grabel L: Hedgehog serves as a mitogen and survival factor during embryonic stem cell neurogenesis. Stem Cells 26: 1097-1108, 2008.

34. Feng Z, Liu L, Zhang C, Zheng T, Wang J, Lin M, Zhao Y, Wang X, Levine AJ and Hu W: Chronic restraint stress attenuates p53 function and promotes tumorigenesis. Proc Natl Acad Sci USA 109: 7013-7018, 2012. 